\title{
Laser printed glass planar lightwave circuits with integrated fiber alignment structures
}

\author{
Desmet, A. ${ }^{\mathrm{a}, \mathrm{b}}$, Radosavljevic, A. ${ }^{\mathrm{b}}$, Missinne, J. ${ }^{\mathrm{a}}$, Van Thourhout, D. ${ }^{\mathrm{b}}$, and Van Steenberge, G. ${ }^{\mathrm{a}}$ \\ ${ }^{a}$ Centre for Microsystems Technology (CMST), imec and Ghent University, Technologiepark \\ 15, 9052 Gent, Belgium \\ ${ }^{b}$ Photonics Research Group, Ghent University - imec, Technologiepark 15, 9052 Ghent, \\ Belgium
}

\begin{abstract}
Femtosecond laser inscription allows straightforward manufacturing of glass planar lightwave circuits such as waveguides, interferometers, directional couplers, resonators and more complex structures. Fiber alignment structures are needed to facilitate communication with the glass planar lightwave circuit. In this study, a technique is described to create optical waveguides and alignment structures in the same laser exposure step. Using an industrial ytterbium-doped $1030 \mathrm{~nm}$ fiber laser pulses of 400 fs were focused into glass with a $0.4 \mathrm{NA}$ objective causing permanent alteration of the material. Depending on laser parameters this modification allows direct writing of waveguides or the creation of channels after exposing the irradiated volumes to an etchant such as $\mathrm{KOH}$. Writing of channels and waveguides with different laser powers, frequencies, polarisations, stage translation speeds and scan densities were investigated in fused silica and borosilicate glass. Waveguides with controlled dimensions were created, as well as etched U-grooves with a diameter of $126 \mu \mathrm{m}$ and a sidewall roughness $\mathrm{R}_{a}$ of $255 \mathrm{~nm}$. Cut back measurements were performed giving a waveguide propagation loss of 1.1 $\mathrm{dB} / \mathrm{cm}$ in borosilicate glass. A coupling loss of $0.7 \mathrm{~dB}$ was measured for a transition between the waveguide and standard single mode fiber at $1550 \mathrm{~nm}$, using index matching liquid. The described technique eliminates active alignment requirements and is useful for many applications such as microfluidic sensing, PLCs, fan-out connectors for multicore fibers and quantum optical networks.
\end{abstract}

Keywords: Ultrafast Laser Inscription, Laser Processing, Packaging, FLICE, glass, waveguides

\section{INTRODUCTION}

Femtosecond laser processing allows 3D modification of dielectric materials such as glass. Using femtosecond pulses, very high peak intensities can be reached which enables multiphoton absorption in the otherwise transparent glass. After the pulses have passed, the electrons relax the absorbed energy to the glass lattice resulting in a permanent modification of the glass. ${ }^{1}$ Because only in the focal volume of the glass these intensities are high enough to drive multiphoton absorption, it is possible to create laser printed waveguides in the bulk of the glass. The observed morphology changes after laser exposure can be summarized by the following 3 structural changes; I) homogeneous modification: ${ }^{2}$ the main mechanisms here appear to be densification by melting and rapidly quenching because of heat accumulation. This mechanism happens mainly for lower pulse energies. II) sub-wavelength nanogratings: ${ }^{3,4}$ formation of nanogratings due to the interference of the laser field with the induced electron plasma wave. This is for intermediate pulse energies. III) voids surrounded by densified area: $:^{5,6}$ When the pulse energy is high, electron-free or electron-rich areas are created, leading to a respectively negative or positive refractive index change. Laser modification is mostly a combination of these 3 changes, but depending on laser parameters specific changes might become dominant.

Further author information: (Send correspondence to A. D.)

A.D.: E-mail: Andres.Desmet@ugent.be, Telephone: +32(0)9 2645370 


\subsection{Inscription}

The ability of ultrafast laser pulses to create optical waveguides in arbitrary 3D paths in glass has been shown by Davis et al in $1996 .^{7}$ Glass chips processed with this inscription technology are perfect candidates for Planar Lightwave Circuits (PLC), and Lab-On-Chip (LOC) applications. The straightforward and fast (for ultrafast lasers with a sufficient repetition rate frequency) nature of this technology also allows rapid prototyping of passive and even active devices suchs as Mach-Zehnder interferometers, ${ }^{8,9}$ Y-Couplers ${ }^{10}$ and Amplifiers. ${ }^{11,12}$

\subsection{FLICE}

Fabrication of mechanical structures in the glass can be achieved by Femtosecond Laser Irradiation followed by Chemical Etching (FLICE). A select volume of glass is first exposed to ultrafast laser pulses and afterwards submerged in an etchant such as $\mathrm{KOH}$ or HF. The irradiated glass has a higher etch rate and it will be removed faster than the non-exposed glass, allowing the creation of trenches.

\subsection{Combining FLICE and Inscription}

Nowadays, active alignment methods are typically used to couple light from optical fibers to planar lightwave circuits (PLC). By defining the volume of glass that needs to be etched and inscribing the waveguides in the same ultrafast laser fabrication process, a trench is created in which an optical fiber can be placed in line with the printed optical waveguide. Leading to facilitated coupling, avoiding cumbersome active alignment.

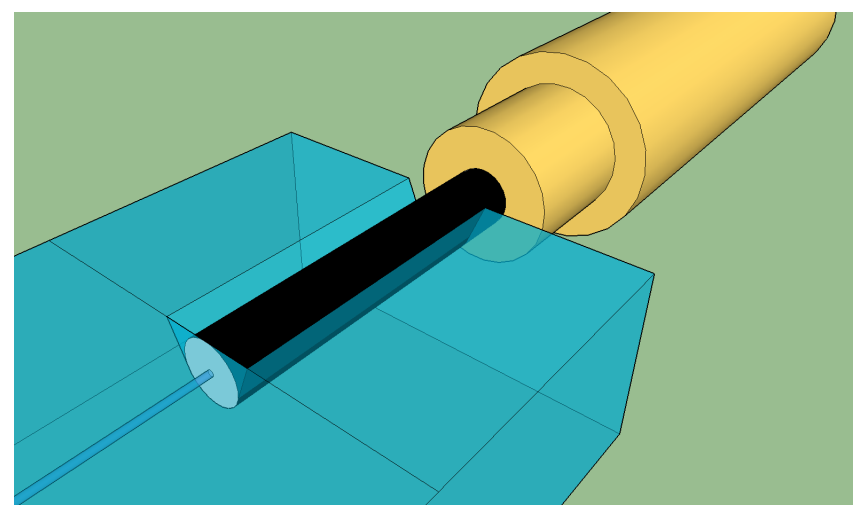

Figure 1: Laser printed glass planar lightwave circuit with integrated fiber alignment structures

\section{METHOD}

The envisioned process is a combination of FLICE and inscription which allows the printing of waveguides and mechanical structures in the same laser exposure step. The etched trench allows the placement of an optical fiber, well-aligned with the glass waveguides, as shown in figure 1.

\subsection{Inscription}

The laser used to inscribe the glass is a fiber chirped pulse amplification (FPCA) laser (Satsuma, Amplitude Systmes) which operates at a wavelength of $1030 \mathrm{~nm}$ and a pulselength of $400 \mathrm{fs}$. The lens used to focus the light is a 0.4 NA ELWD (extra-long working distance) microscope objective from Nikon. The type of glass used is borosilicate glass from Schott (D263 T). A motorized stage allows movement of the sample with respect to the laser in the horizontal plane. Laser powers around $300 \mathrm{~mW}$, a repetition rate of $1 \mathrm{MHz}$ and translation speeds of motorized stage ranging from $0.5 \mathrm{~mm} / \mathrm{s}$ to $10 \mathrm{~mm} / \mathrm{s}$ are used. By bringing the focusing objective closer with respect to the sample, the waveguides and trenches can be printed deeper. After the laser process, the edges of the glass are diced, since when writing near the edge of the glass, the beam will not be focused uniformly in the glass any more leading to different inscription regimes. The edge effects get worse for larger inscription depths and lower NA. After the dicing step, the sidewalls of the glass were polished to decrease coupling loss. 
The average surface roughness after polishing is $70 \mathrm{~nm}$. After the samples were diced and polished, they are placed in a horizontal optical set-up for loss measurements and mode analysis. A SMF-28 fiber is aligned at the in-coupled facet with the waveguide. Then, light at $635 \mathrm{~nm}$ and $1550 \mathrm{~nm}$ is coupled to the fiber. At the out-coupling facet the light is collected in another SMF-28 fiber which is coupled to a power meter. Then the glass is repeatedly diced and polished again to get the waveguides loss for decreasing lengths. By measuring the power transmitted at each different length, propagation losses can be calculated.

\subsection{FLICE}

The laser process for FLICE is similar as for inscription, but a different laser scheme is used to irradiate a larger volume, compatible with the dimensions of an optical fiber $(125 \mu \mathrm{m})$. To etch away sufficient material multiple lines immediately next to each are scanned with the laser with horizontal pitches between $0.5 \mu \mathrm{m}$ and $5 \mu \mathrm{m}$, and vertical pitches between $1 \mu \mathrm{m}$ and $10 \mu \mathrm{m}$. Laser powers vary from $100 \mathrm{~mW}$ to $300 \mathrm{~mW}$, depending on the translation speed which ranges from $10 \mathrm{~mm} / \mathrm{s}$ to $200 \mathrm{~mm} / \mathrm{s}$ with frequencies between $100 \mathrm{kHz}-1000 \mathrm{kHz}$. The glass used here is fused silica. After the glass sample has been irradiated by the laser, the sample is submerged in a $30 \% \mathrm{KOH}$ solution at a temperature of $80^{\circ}$ with etch times up to 8 hours. Analysis is done by diascopic and episcopic camera images together with a white light optical interferometer (WYKO NT3300) for roughness measurements.

\section{RESULTS AND DISCUSSION}

\subsection{Waveguide Inscription}

\subsubsection{Loss measurements}

Six samples of $10 \mathrm{~cm}$ long borosilicate glass were inscribed with the laser focused $100 \mu \mathrm{m}$ below surface. The glass is irradiated at a stage translation speed of $1 \mathrm{~mm} / \mathrm{s}$, a pulse repetition frequency of $1 \mathrm{MHz}$ and 6 different powers ranging from $0.288 \mathrm{~W}$ to $0.3 \mathrm{~W}$. To avoid edge-effects, $0.5 \mathrm{~cm}$ was removed from each side by dicing and consecutively polished. After this cutback loss measurement was performed. Losses for the inscribed waveguides are shown in figure 2. Linear fitting was done; the slope stands for the propagation losses in $\mathrm{dB}$ per $\mathrm{cm}$ and the intersection with Y-axis gives the in- and outcoupling losses in dB. The best results are for an inscription power of $0.291 \mathrm{~W}$ with a propagation loss of $1.1 \mathrm{~dB}$ loss per $\mathrm{cm}$ and a coupling loss of $4.6 \mathrm{dBm}$ corresponding to an in-and outcoupling loss of $2.3 \mathrm{~dB}$. For the other powers, a loss of $1.3 \mathrm{~dB} / \mathrm{cm}, 1.51 \mathrm{~dB} / \mathrm{cm}, 1.39 \mathrm{~dB} / \mathrm{cm}, 1.3$ $\mathrm{dB} / \mathrm{cm}$ and $1.6 \mathrm{~dB} / \mathrm{cm}$ is measured for $0.3 \mathrm{~W}, 0.298 \mathrm{~W}, 0.295 \mathrm{~W}, 0.29 \mathrm{~W}$ and $0.288 \mathrm{~W}$ respectively. Coupling losses fluctuate around $4 \mathrm{~dB}$ with an outlier at $2.7 \mathrm{~dB}$ for $0.288 \mathrm{~W}$. These higher coupling losses can be explained by 2 times (in- and outcoupling) the mismatch between the Mode Field Diameter (MFD) of the the inscribed glass which is around $12 \mu \mathrm{m}$ to $16 \mu \mathrm{m}$ and the MFD of the SMF-28 optical fiber which is $10.4 \mu \mathrm{m}$ at $1550 \mathrm{~nm}$. 

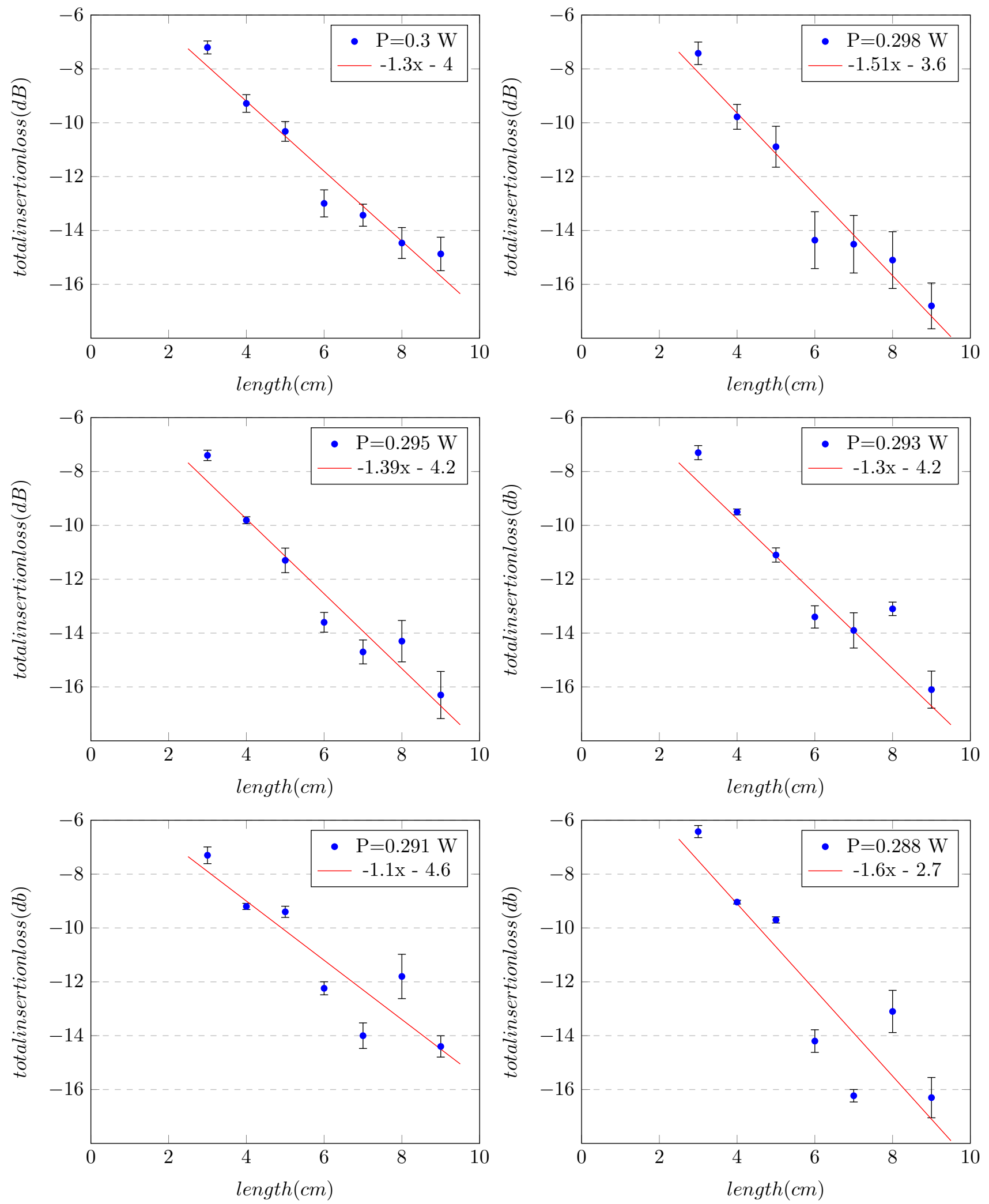

Figure 2: Losses versus Length 
Figure $3 \mathrm{a}$ and $3 \mathrm{~b}$ shows the mode profile of a printed waveguide at $635 \mathrm{~nm}$ and $1550 \mathrm{~nm}$ respectively measured in the near-field. The mode at $635 \mathrm{~nm}$ was measured with a beam profilometer (Spiricon SP620U beam) with a 40x lens and visualised with the accompanying software. Each pixel of the camere has a $4.4 \mu \mathrm{m}$ size, which leads to a MFD of $7 \mu \mathrm{m}$. Figure 3b was measured with an infra-red camera by Xenics, after expansion by a x100 lens. The pixel size of the camera is $30 \mu \mathrm{m}$, leading to a MFD of $12.4 \mu \mathrm{m}$ at $1550 \mathrm{~nm}$. This printed waveguide is single mode, since even when in-coupling conditions were changed, to try and couple into other modes, the mode stayed one bright spot.

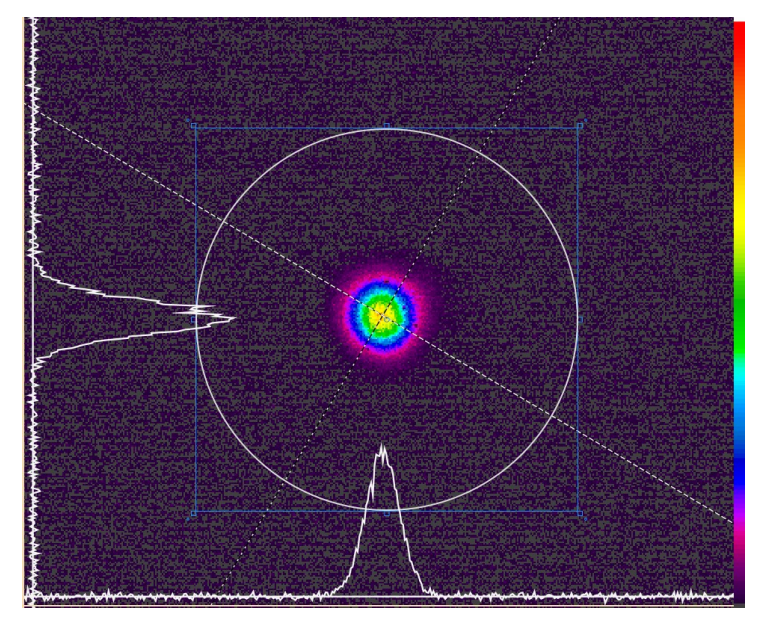

(a)

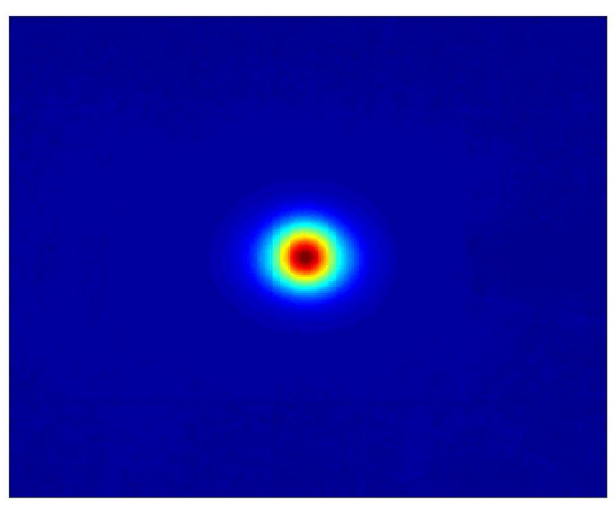

(b)

Figure 3: Mode profiles of a waveguide inscribed at $0.3 \mathrm{~W}, 1 \mathrm{MHz}, 1 \mathrm{~mm} / \mathrm{s}$ a at $635 \mathrm{~nm}, \mathrm{~b}$ at $1550 \mathrm{~nm}$.

\subsubsection{Alignment tolerance}

In this section the power transmission with a rising misalignment between the fiber and printed waveguide is simulated. Using the inverse Helmholtz technique described in $^{13}$ the refractive index distribution of the glass is calculated, using the measured mode profile. Starting from a constant refractive index of 1.55 for the nonmodified glass, a peak refractive index of 1.558 is calculated, which corresponds to a $0.52 \%$ peak refractive index change. Based on these results, propagation through the inscribed waveguides structures is simulated using fimmwave. 


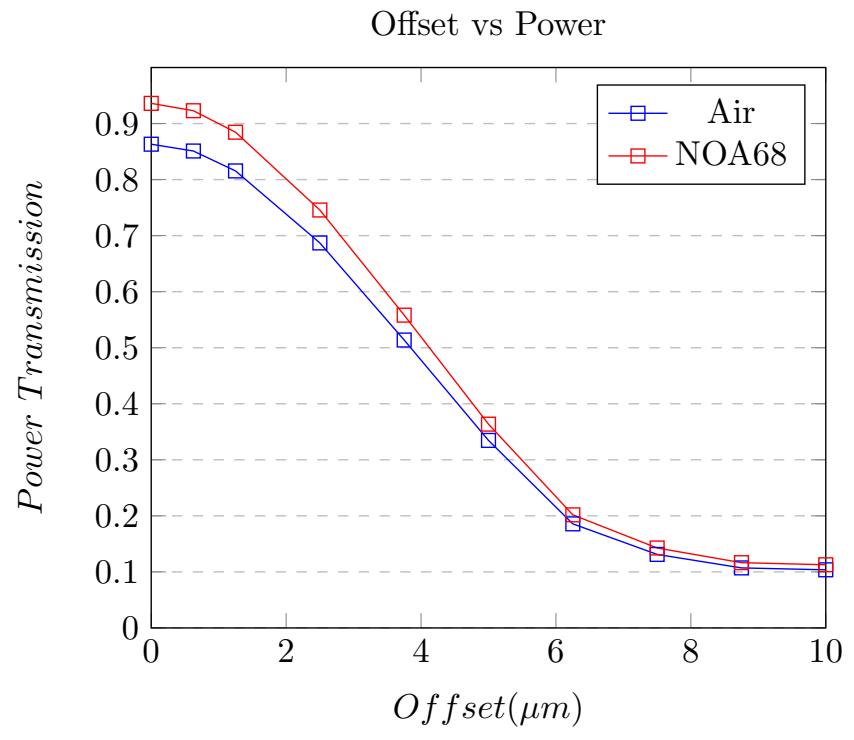

Figure 4: Alignment tolerance

Figure 4 shows the simulated power transmission for a rising degree of misalignment between an SMF-28 optical fiber and an inscribed waveguide. A 1 micron distance between the end facet of the fiber and the start of the glass facet is assumed. For the blue curve, this gap is filled with air, with a refractive index of 1 , for the red curve, the gap is filled with NOA 68, which has a refractive index of 1.54 . These simulations show that for a lateral misalignment under $4 \mu \mathrm{m}$, the power transmission is still over $50 \%$, or a loss of less than $3 \mathrm{~dB}$, and for a lateral misalignment under $2 \mu \mathrm{m}$, the power transmission is over $80 \%$, or a loss of less than $1 \mathrm{~dB}$. If a channel can thus be etched in front of the printed waveguide with an accuracy under $2 \mu \mathrm{m}$, losses will be less than $1 \mathrm{db}$. These simulations only include losses due to mode mismatch and misalignment, scattering losses due to roughness and due to an angle between the two waveguides are not included.

\subsection{FLICE}

Since FLICE is a two step process; the laser step followed by the etching step, there are many parameters that must be controlled. The etching solution and temperatures are fixed at a $30 \% \mathrm{KOH}$ solution at $80^{\circ} \mathrm{C}$. Etching time depends on laser parameters, such as polarisation, power and translation speed. To be able to irradiate a selected volume, multiple lines will be scanned which leads to a horizontal and vertical pitch between the lines.

\subsubsection{Parameter Study}

The orientation of the laser polarisation plays an important role in FLICE since it determines the orientation of the nanogratings that are created. Due to interference between the laser field with the induced electron plasma wave, nanogratings are formed with an orientation perpendicular to the (linear laser) polarisation. When these nanogratings are along the to be etched channel, it greatly increases etching rates, as can be seen in figure 5 and figure 6. Samples were irradiated with the laser at a frequency of $500 \mathrm{kHz}$, power $140 \mathrm{~mW}$ and a stage translation speed of $10 \mathrm{~mm} / \mathrm{s}$. The lateral pitch of the individual lines is $1 \mu \mathrm{m}$, and the vertical pitches are 6 $\mu \mathrm{m}, 8 \mu \mathrm{m}$ and $10 \mu \mathrm{m}$. Each group contains 5 channels with a sweep in laser repetition where the first channel in each group is scanned with the laser once and the fifth channel five times. Repeating the laser process for these parameters multiple times increases the etching rates, as can be seen Figure 5 shows etching after laser irradiation with polarisation along the writing direction, figure 6 shows etching after laser irradiation with polarisation perpendicular to the writing direction. Figure 5 shows that after 30 min only trenches with a z-pitch of $6 \mu \mathrm{m}$ have some visible etching, with $100 \mu \mathrm{m}$ etching on the fifth line. After 90 minutes up to $325 \mu \mathrm{m}$ is etched, this corresponds with an etch rate of $200 \mu \mathrm{m} / \mathrm{h}$. For figure 6 the $2 \mathrm{~mm}$ long channels were completely etched away, which is an etch rate of $>4000 \mu \mathrm{m} / \mathrm{h}$. Because of this high etch rate, the uniformity of the channels is lost. Furthermore etching with laser polarisation perpendicular to the writing direction leads to more lateral 
etching and thus increases the width of the channels. For the Z-pitches of $6 \mu \mathrm{m}$ and $8 \mu \mathrm{m}$, the lateral etching has lead to the connection of certain channels. For a Z-pitch of $10 \mu \mathrm{m}$, the lateral etching is confined to the individual channels. These effects might be explained due to formation of nanogratings perpendicular to the laser polarization.

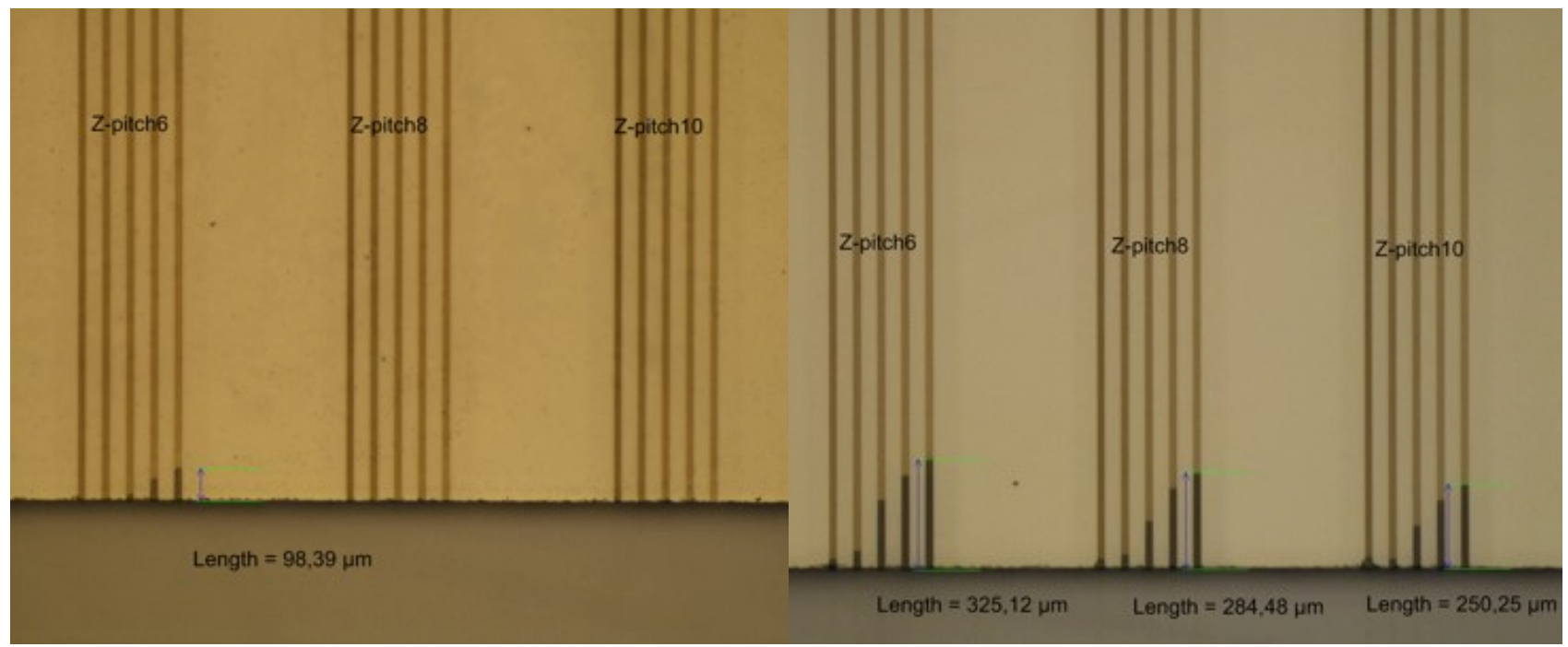

(a)

(b)

Figure 5: Top view microscope image of channels etched by FLICE with laser polarisation along the writing direction: (a) after etching for 30 minutes, (b) after etching for 90 minutes

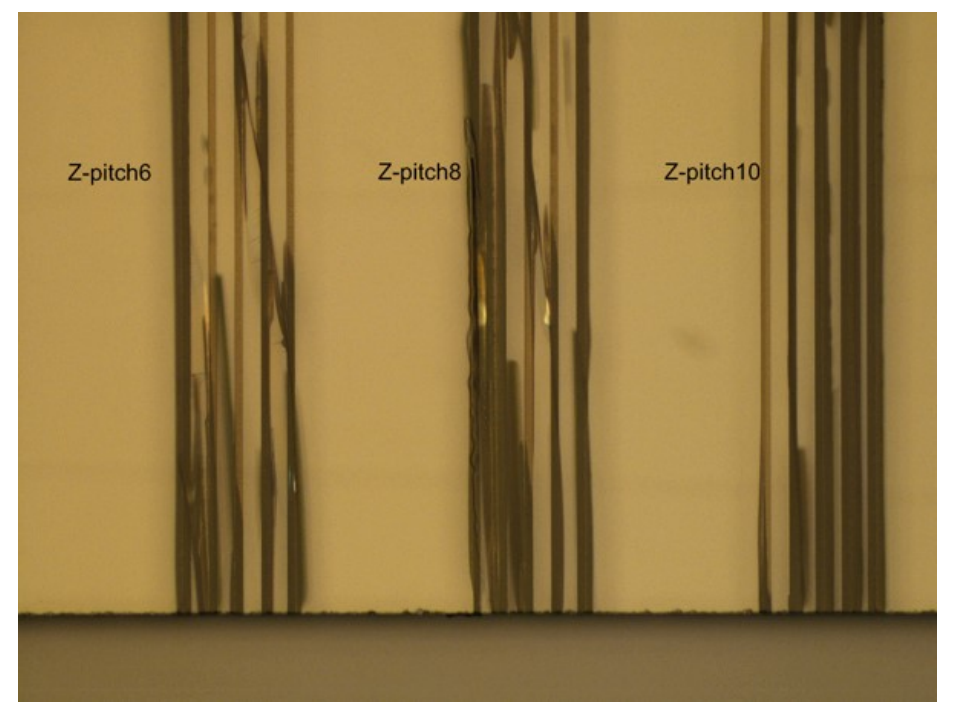

Figure 6: Top view microscope image of channels etched by FLICE with laser polarisation perpendicular to the writing direction: etching for 30 minutes

\subsubsection{Cross-section}

Figure $7 \mathrm{a}$ and $7 \mathrm{~b}$ show cross-sections of etched profiles. A $2 \mu \mathrm{m}$ y-pitch and a $6 \mu \mathrm{m}$ z-pitch was used for figure $7 \mathrm{a}$, with $\mathrm{x}$ the direction along the channel and $\mathrm{y}$ the width of the channel. Figure $7 \mathrm{~b}$ was lasered with a $1 \mu \mathrm{m}$ y-pitch and a $2 \mu \mathrm{m}$ z-pitch and shows cracks after etching. Using a different laser scheme with higher pitches 
might avoid cracks. By using the correct amount of lines, the form of the selected volume to be removed can be controlled so that the placement of an optical fiber is possible.

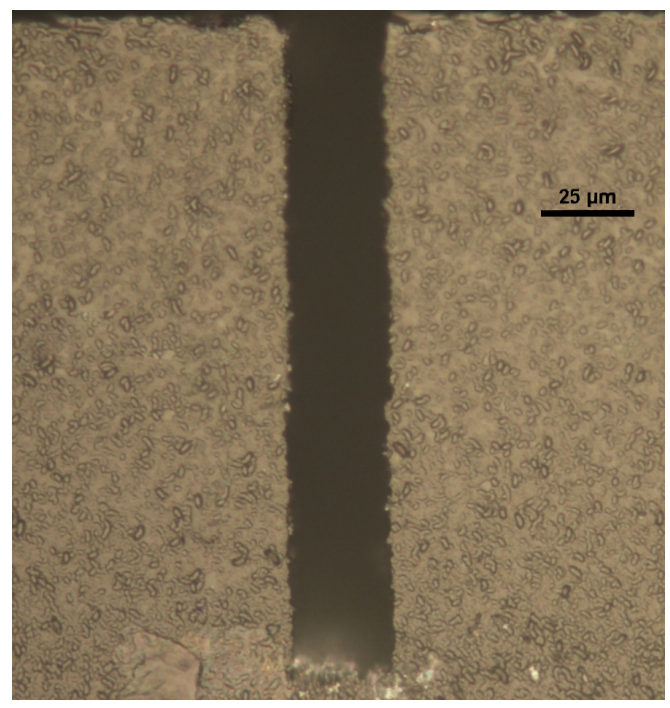

(a)

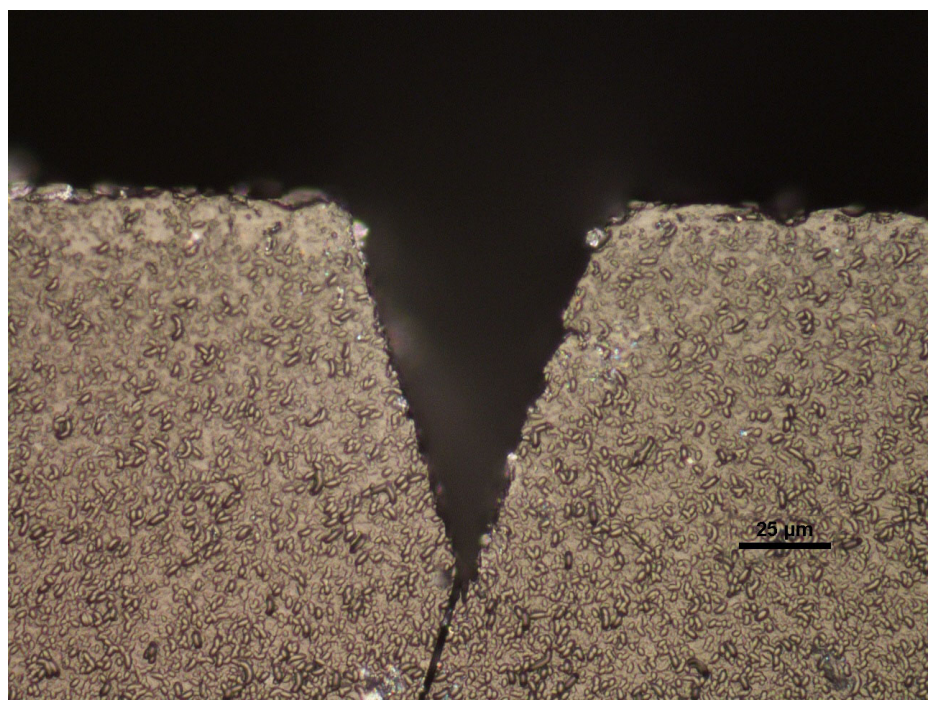

(b)

Figure 7: Cross-sections of a rectangulary etched profile (a), and a V-groove (b). Both figures are the result of fused silica being lasered at $750 \mathrm{kHz}, 200 \mathrm{~mm} / \mathrm{s}, 250 \mathrm{~mW}$ and were consecutevely etched in a $30 \% \mathrm{KOH}$ solution for 4 hours, but with different laser schemes.

\subsubsection{Roughness}

Since form can be controlled with choosing a correct pitch and number of lines, the next thing to check is roughness. Figure 8 shows a roughness measurement of a sidewall after FLICE measured with a WYKO white light interferometer. The sample was irradiated with the laser at a frequence of $750 \mathrm{kHz}$, a power of $250 \mathrm{~mW}$ and stage movement speed of $200 \mathrm{~mm} / \mathrm{s}$. The sample was consecutively etched for 4 hour by a $30 \% \mathrm{KOH}$ solution at a temperature of $80^{\circ} \mathrm{C}$. The average roughness $\mathrm{Ra}$ is $255 \mathrm{~nm}$, while the RMS roughness $\mathrm{Rq}$ is $328 \mathrm{~nm}$ for a $120 \mu \mathrm{m} \times 92 \mu \mathrm{m}$ area, which is low enough for alignment purposes.

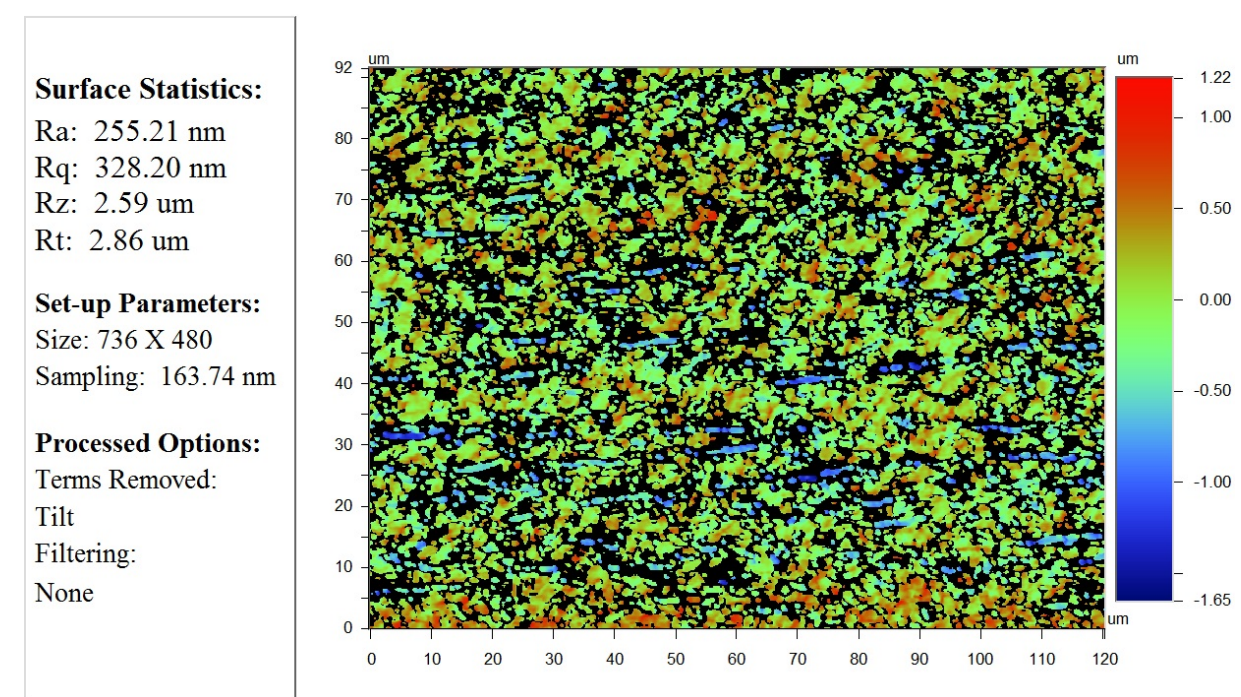

Figure 8: Roughness measurement of the sidewall of a channel defined in fused silica using the FLICE process. 


\section{CONCLUSION}

A method to inscribe waveguides in glass in combination with FLICE for mechanical alignment structures is proposed. This allows for immediate alignment between an optical fiber and a printed waveguide in glass, facilitating the in- and out-coupling alignment process. Waveguides inscribed at a frequency of $1 \mathrm{MHz}$, a power of $0.3 \mathrm{~W}$ and a stage translation speed of $1 \mathrm{~mm} / \mathrm{s}$ were presented with losses of $1.1 \mathrm{~dB} / \mathrm{cm}$. Using these waveguides PLC's with passive devices suchs as Mach-Zehnder interferometers ${ }^{8,9}$ and Y-Couplers ${ }^{10}$ can be made. Using FLICE, profiles can be etched away in a U- or V-shape to allow placement of a fiber. Glass is first exposed to laser irriadiation and consecutively etched in a $30 \% \mathrm{KOH}$ solution at $80^{\circ}$ at an etch rate $>2 \mathrm{~mm} / \mathrm{h}$. A sidewall roughness Ra of $255 \mathrm{~nm}$ is measured. Utilizing an index matched adhesive, the optical fiber can be fixed to a glass chip with limited in- and out-coupling losses. Simulations show that the loss caused by an offset of $2 \mu \mathrm{m}$ is less than $1 \mathrm{~dB}$. Loss due to mode mismatch can be reduced by sufficiently matching the mode of the waveguides with that of the optical fiber. This method allows for easy pigtailing of glass planar lightwave circuits without the need of any cumbersome active alignment steps.

\section{ACKNOWLEDGMENTS}

The authors would like to acknowledge the financial support from the Special Research Fund of Ghent University (BOF14/GOA/034 project), and from the Hercules Foundations Flanders (ZW09-01: APPLIE4MOS).

\section{REFERENCES}

[1] Eaton, S., Contrasts in thermal diffusion and heat accumulation effects in the fabrication of waveguides in glasses using variable repetition rate femtosceond laser, $\mathrm{PhD}$ thesis, University of Toronto (2008).

[2] Miura, K., Qiu, J., Inouye, H., Mitsuyu, T., and Hirao, K., "Photowritten optical waveguides in various glasses with ultrashort pulse laser," 71, 3329-3331 (12 1997).

[3] Sudrie, L., Franco, M., Prade, B., and Mysyrowicz, A., "Writing of permanent birefringent microlayers in bulk fused silica with femtosecond laser pulses," Optics Communications 171(4), 279 - 284 (1999).

[4] Hnatovsky, C., S. Taylor, R., Pattathil, R., Simova, E., R. Bhardwaj, V., M. Rayner, D., and B. Corkum, P., "Pulse duration dependence of femtosecond-laser-fabricated nanogratings in fused silica," 87, 014104014104 (06 2005).

[5] N. Glezer, E. and Mazur, E., "Ultrafast-laser driven micro-explosions in transparent materials," 71, 882-884 (08 1997).

[6] Glezer, E. N., Milosavljevic, M., Huang, L., Finlay, R. J., Her, T.-H., Callan, J. P., and Mazur, E., "Threedimensional optical storage inside transparent materials," Opt. Lett. 21, 2023-2025 (Dec 1996).

[7] Davis, K., Miuara, K., and Hirao, K., "Writing waveguides in glass with a femtosecond laser," Optics Letters 21, 1729-1731 (1996).

[8] Florea, C. and Winick, K., "Fabrication and characterization of photonic devices directly written in glass using femtosecond laser pulses," Journal of lightwave technology 21, 246-253 (2003).

[9] Minoshima, K., Kowalevicz, A. M., Hartl, I., Ippen, E. P., and Fujimoto, J. G., "Photonic device fabrication in glass by use of nonlinear materials processing with a femtosecond laser oscillator," Opt. Lett. 26, 15161518 (Oct 2001).

[10] Homoelle, D., Wielandy, S., Gaeta, A. L., Borrelli, N. F., and Smith, C., "Infrared photosensitivity in silica glasses exposed to femtosecond laser pulses," Opt. Lett. 24, 1311-1313 (Sep 1999).

[11] Florea, C., Winick, K., Sikorski, Y., Said, A., and Bado, P., "Optical waveguide amplifier in nd-doped glass written with near-ir femtosecond laser pulses," in [Conference on Lasers and Electro-Optics], Conference on Lasers and Electro-Optics, CMX5, Optical Society of America (2000).

[12] Osellame, R., Taccheo, S., Marangoni, M., Ramponi, R., Laporta, P., Polli, D., Silvestri, S. D., and Cerullo, G., "Femtosecond writing of active optical waveguides with astigmatically shaped beams," J. Opt. Soc. Am. B 20, 1559-1567 (Jul 2003).

[13] Mansour, I. and Caccavale, F., "An improved procedure to calculate the refractive index profile from the measured near-field intensity," Journal of lightwave technology 14, 423-428 (1996). 